\title{
CDKN2A-p16 Positive
}

National Cancer Institute

\section{Source}

National Cancer Institute. CDKN2A-p16 Positive. NCI Thesaurus. Code C129258.

Indicates that CDKN2A-p16 expression has been detected in a sample. 